\title{
Recurrence risk for germinal mosaics revisited
}

\author{
Martin A van der Meulen, Meine J P van der Meulen, Gerard J te Meerman
}

\begin{abstract}
A formula to calculate recurrence risk for germline mosaicism published by Hartl in 1971 has been updated to include marker information. For practical genetic counselling new, more elaborate tables are given.
\end{abstract}

(f Med Genet 1995;32:102-104)

In 1974 Murphy et $a l^{1}$ concluded "From the clinical standpoint the main implication is that it provides reassurance that, in any realistic size of family, ignoring the effect of gonadal mosaicism will have little effect on the estimate of the risk for the next child". This paper has been referred to many times in papers about mosaicism. A paper by $\mathrm{Hartl}^{2}{ }^{2}$ entitled "Recurrence risk for germinal mosaics", gives formulae to calculate recurrence risks, correcting for the number of affected and unaffected sibs. This paper will discuss the impact of modern molecular genetic techniques on the recurrence risk for gonadal mosaicism.

\section{The theory}

In all humans some degree of mosaicism is the norm for the more common genetic disorders, since the mutation rate multiplied by the number of mitoses necessary to form the 5-7 million $^{3}$ or 8 million oocytes ${ }^{4}$ in females and the many more spermatozoa in males is much higher than one. In mosaics distinction has to be made between somatic and germline mosaics. In somatic mosaics the germ stem cell is mutated. This mutation happens in one of the 46 chromosomes $(2 n)$ in a mitosis between fertilisation and the mitoses leading to the germ stem cell. In somatic mosaics all gonadal cells before meiosis will have the same mutation on one chromosome, and because of the meiosis in which a 2 n chromosome cell is split into two cells with $\mathrm{n}$ chromosomes, there is a $\mathbf{5 0 \%}$ recurrence risk. In germline mosaics a fraction of the gonadal cells will have a mutation. This is dependent on the gonadal generation in which the mutation occurred, since all descendent cells of the mutated cell are carriers of the mutation. For a more detailed description of this model see Murphy et al. ${ }^{1}$ The recurrence risk is dependent on the gonadal generation in which the mutation occurred to a maximum of $50 \%$ (first gonadal generation mutation).

The recurrence risk owing to germline mosaicism can be explained through the development of all $2^{\mathrm{n}}$ oocytes from one healthy wild type cell. In $2^{\mathrm{n}}, \mathrm{n}$ is the number of cell generations to get from one healthy cell to the total amount of oocytes. Hartl ${ }^{2}$ showed that more complex models than this simple model do not change recurrence risks as long as the number of gonadal generations is high enough. If we assume that during gametogenesis the probability of mutation in each gonadal generation, conditional on the occurrence of a mosaic genotype, is the same for all cell generations, the recurrence risk owing to germline mosaicism, only taking into account the information that one parent is a mosaic, can be calculated from the number of cell generations $\mathrm{n}$ to be

$$
\text { Recurrence risk }=\sum_{i=1}^{n} \frac{1}{n} *(2)^{-i} \approx \frac{1}{n} .
$$

According to Hartl, ${ }^{2}$ in human females the effective number of gonadal generations $\mathrm{n}$ is $10-12$. This will give a recurrence risk of $8 \cdot 3 \%$. If, however, it is assumed that during fetal life the number of oocytes reaches 5-7 million, ${ }^{3} n$ has to be at least 22 in females. This gives a recurrence risk of $4.5 \%$. In males $n$ has to be at least $30 .{ }^{34}$ Note that the recurrence risk is independent of the mutation rate. The recurrence risk is conditional on the fact that one of the parents is a germinal mosaic, owing to a single mutation.

Bakker $e t \mathrm{al}^{5}$ estimated recurrence risks for $\mathrm{X}$ linked Duchenne muscular dystrophy mosaics from empirical data to be $7 \%$. Since families are not collected at random, proper correction for ascertainment bias is essential. Proper ascertainment bias correction is only possible if the correct model is known, which is rarely the case. ${ }^{6}$ Prenatal diagnoses based on $\mathrm{X}$ haplotype information either leads to the exclusion of the at risk $\mathrm{X}$ chromosome or a $14 \%$ recurrence risk for male pregnancies. Van Essen et al give an overview of the pooled results from 25 European centres.

$\mathrm{Hartl}^{2}$ gives a formula to calculate the recurrence risk from the number of affected and unaffected sibs. He does not, not surprisingly in 1971, correct for marker information, confirming that sibs actually inherited the at risk chromosome from their mosaic parent. Of course, his calculations are still valid, if there are no marker data available, for instance because the location of the disease is unknown or because there is no genetic material from affected sibs, so the at risk chromosome can not be determined. To include marker information in recurrence risk calculations the formula has to be extended to take in typed and untyped sibs, making more accurate risk calculations possible.

If we define the percentage of affected chromosomes $\mathbf{R}$, the following classes of sibs can be distinguished. 
Table 1 Recurrence risk for gonadal mosaics with one affected child $(a=1)$ and a variable number of untyped unaffected children (b) and typed unaffected children with the at risk chromosome (c)

\begin{tabular}{|c|c|c|c|c|c|c|}
\hline \multirow[t]{2}{*}{ Recurrence risk $(a=1)$} & & \multicolumn{5}{|c|}{ No of typed unaffected children with the at risk chromosome (c) } \\
\hline & & 0 & 1 & 2 & 3 & 4 \\
\hline $\begin{array}{l}\text { No of untyped } \\
\text { unaffected children (b) }\end{array}$ & $\begin{array}{l}0 \\
1 \\
2 \\
3 \\
4\end{array}$ & $\begin{array}{l}0.048 \\
0.033 \\
0.025 \\
0.019 \\
0.016\end{array}$ & $\begin{array}{l}0.018 \\
0.015 \\
0.013 \\
0.012 \\
0.011\end{array}$ & $\begin{array}{l}0.013 \\
0.012 \\
0.010 \\
0.009 \\
0.009\end{array}$ & $\begin{array}{l}0.010 \\
0.009 \\
0.008 \\
0.008 \\
0.007\end{array}$ & $\begin{array}{l}0.008 \\
0.008 \\
0.007 \\
0.007 \\
0.006\end{array}$ \\
\hline
\end{tabular}

Table 2 Recurrence risk for gonadal mosaics with two affected children $(a=2)$ and a variable number of untyped unaffected children (b) and typed unaffected children with the at risk chromosome (c)

\begin{tabular}{|c|c|c|c|c|c|c|}
\hline \multirow{2}{*}{\multicolumn{2}{|c|}{ Recurrence risk $(a=2)$}} & \multicolumn{5}{|c|}{ No of typed unaffected children with the at risk chromosome (c) } \\
\hline & & 0 & 1 & 2 & 3 & 4 \\
\hline $\begin{array}{l}\text { No of untyped } \\
\text { unaffected children (b) }\end{array}$ & $\begin{array}{l}0 \\
1 \\
2 \\
3 \\
4\end{array}$ & $\begin{array}{l}0 \cdot 333 \\
0 \cdot 286 \\
0 \cdot 240 \\
0 \cdot 200 \\
0 \cdot 168\end{array}$ & $\begin{array}{l}0 \cdot 143 \\
0 \cdot 133 \\
0 \cdot 124 \\
0 \cdot 115 \\
0 \cdot 107\end{array}$ & $\begin{array}{l}0 \cdot 120 \\
0 \cdot 111 \\
0 \cdot 103 \\
0 \cdot 096 \\
0.089\end{array}$ & $\begin{array}{l}0.100 \\
0.093 \\
0.087 \\
0.081 \\
0.075\end{array}$ & $\begin{array}{l}0.084 \\
0.079 \\
0.074 \\
0.069 \\
0.065\end{array}$ \\
\hline
\end{tabular}

Table 3 Recurrence risk for gonadal mosaics with three affected children $(a=3)$ and a variable number of untyped unaffected children (b) and typed unaffected children with the at risk chromosome (c)

\begin{tabular}{lllllll}
\hline Recurrence risk $(a=3)$ & & \multicolumn{5}{c}{ No of typed unaffected children with the at risk chromosome $(c)$} \\
\cline { 3 - 7 } & & 0 & 1 & 2 & 3 & 4 \\
\hline No of untyped & & & & & & \\
unaffected children (b) & 0 & 0.429 & $0 \cdot 200$ & $0 \cdot 183$ & $0 \cdot 164$ & $0 \cdot 145$ \\
& 1 & 0.400 & $0 \cdot 194$ & $0 \cdot 175$ & $0 \cdot 156$ & $0 \cdot 138$ \\
& 2 & 0.366 & $0 \cdot 187$ & $0 \cdot 168$ & $0 \cdot 149$ & $0 \cdot 131$ \\
& 3 & 0.328 & $0 \cdot 179$ & $0 \cdot 160$ & $0 \cdot 141$ & $0 \cdot 124$ \\
& 4 & $0 \cdot 290$ & $0 \cdot 172$ & $0 \cdot 153$ & $0 \cdot 134$ & $0 \cdot 118$ \\
\hline
\end{tabular}

Table 4 Recurrence risk for gonadal mosaics with four affected children $(a=4)$ and a variable number of untyped unaffected children (b) and typed unaffected children with the at risk chromosome (c)

\begin{tabular}{|c|c|c|c|c|c|c|}
\hline \multirow{2}{*}{ Recurrence risk $(a=4)$} & & \multicolumn{5}{|c|}{ No of typed unaffected children with the at risk chromosome (c) } \\
\hline & & 0 & 1 & 2 & 3 & 4 \\
\hline $\begin{array}{l}\text { No of untyped } \\
\text { unaffected children (b) }\end{array}$ & $\begin{array}{l}0 \\
1 \\
2 \\
3 \\
4\end{array}$ & $\begin{array}{l}0.467 \\
0.452 \\
0.431 \\
0.406 \\
0.375\end{array}$ & $\begin{array}{l}0 \cdot 226 \\
0 \cdot 222 \\
0 \cdot 218 \\
0 \cdot 214 \\
0 \cdot 209\end{array}$ & $\begin{array}{l}0 \cdot 216 \\
0 \cdot 211 \\
0 \cdot 206 \\
0 \cdot 200 \\
0 \cdot 195\end{array}$ & $\begin{array}{l}0 \cdot 203 \\
0 \cdot 197 \\
0 \cdot 191 \\
0 \cdot 185 \\
0 \cdot 178\end{array}$ & $\begin{array}{l}0 \cdot 188 \\
0 \cdot 181 \\
0 \cdot 175 \\
0 \cdot 168 \\
0 \cdot 161\end{array}$ \\
\hline
\end{tabular}

Untyped sibs:

Affected: $100 \%$ chance of having the risk chromosome, $\mathrm{R} \%$ of the sibs;

Unaffected: $(50-\mathrm{R}) \%$ chance of having the risk chromosome, $50 \%$ of having the not risk chromosome. Total (100-R)\% of the sibs.

Typed sibs: first of all to be used to determine if the grandmaternal or the grandpaternal chromosome is the mosaic chromosome.

Affected: has the at risk chromosome, informative to increase recurrence risk, $\mathrm{R} \%$ of the sibs.

Unaffected: having the at risk chromosome, informative to decrease a priori risk, $(50-\mathrm{R}) \%$ of the sibs; not having the at risk chromosome, not informative, except for determination on which chromosome the mutation occurred (grandpaternal or grandmaternal), $50 \%$ of the sibs.

From all the classes mentioned above, the three informative classes have to be included in a risk calculation: the affected (A), the untyped unaffected (B), and the typed unaffected with the at risk chromosome (C). The unaffected sibs who are typed and have the non-mosaic chromosome are not informative, because they do not provide information about the frequency of the mosaic chromosome and therefore of the recurrence risk $\mathbf{R}$.

Extending the recurrence risk formula as given by $\mathrm{Hartl}^{2}$ with the third class of typed unaffected children with the at risk chromosome leads to the straightforward Bayesian formula, where $P(A / F)$ is the probability that the next child born to a mosaic parent is affected, given the distribution $F$ of the number of affected children (a), untyped unaffected children (b), and typed unaffected children with the risk chromosome (c). Details of derivation of the formula are shown in the appendix.

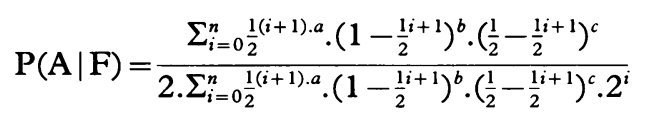

\section{Results}

Since the formula is quite complex, recurrence risks are calculated and shown in tables 1 to 4 for the situations which appear most often in practical genetic counselling. The recurrence risks are calculated given at least one affected child. Wijsman ${ }^{3}$ and Edwards ${ }^{4}$ estimated that the number of gonadal generations $\mathrm{n}$ must be approximately 23 in females and approximately 30 in males. Recurrence risks are almost constant if $\mathrm{n}$ is greater or equal to 20 and because we do not want to underestimate the recurrence risk, $\mathrm{n}$ is set to 20 . The risks in the last column of table 1 from $\mathrm{Hartl}^{2}$ can then be found in the tables. The risks in the tables are the recurrence risks for the next child given the pedigree with at least one affected child and no chromosomal information on the "newborn". Prenatal information on the inherited chromosome of the "newborn" reduces the risk to 0 if the not at risk chromosome is encountered or doubles the risk as stated in the tables otherwise.

\section{Discussion}

One of the major problems in risk calculation for germline mosaics is the impact on the recurrence risk in comparison to the impact of somatic mosaics and, in $\mathrm{X}$ linked recessive cases, the carrier probability of the mother. In Duchenne muscular dystrophy (X linked) onethird of the cases are thought to be the result of a new mutation. ${ }^{89}$ If parents are non-carriers, as can be seen from their phenotype in dominant cases, the mutation must be either somatic or germinal. Although it is unclear how to divide probabilities over somatic and germline mosaics, the impact of somatic mosaics is higher because in this case $50 \%$ of the children are affected against approximately $5 \%$ in germline mosaics. However, one of the advantages of modern genetic technology is the ability to determine which chromosome is inherited from the parents. This way it can be proven that one of the parents is a germline mosaic if an affected and an unaffected child, carrying the same chromosome from one of the parents, is en- 
countered. Germline mosaicism is proven if at least two children have the same genotype, but different phenotype (affected and unaffected). Still, care has to be taken to exclude phenocopies, mutation in the affected child itself leading to somatic mosaicism, incomplete penetrance, or intragenic recombinations.

The formula presented in this paper has an equal conditional probability of mutation in each gonadal generation. Passos-Bueno et $a l^{10}$ concluded that different mosaicism frequencies for proximal and distal DMD mutations exist. The proximal mutations in the DMD families are thought to have a higher recurrence risk through occurring very early in embryonal development, therefore increasing the proportion of mutated cells. The formula can be extended to take the different mutation probabilities of the different gonadal generations into account as soon as more information is available.

In practical genetic counselling, there is often no chromosomal material available from an affected diseased child. Since it is therefore unknown which chromosome of the parents is the mosaic chromosome, special care must be taken. If, for instance, there is a pedigree available with an $\mathrm{X}$ linked disease, one affected son genotype unknown, one unaffected son with the mother's paternal chromosome and two sons with the mother's maternal chromosome, the situation can be analysed as:

(1) Treating all sons as unknown genotype, since it is not known what the at risk genotype is. In table 1, one affected and three unaffected sons can be found with a recurrence risk of $1.9 \%(A=1, X=3, G=0)$.

(2) Analysing the situation in two steps: if the affected son has the mother's father's chromosome, the recurrence risk is, given one unaffected brother (table $1, A=1, X=0, G=1$ ) $1 \cdot 8 \%$; if the affected son has the mother's mother's chromosome, the recurrence risk is, given two unaffected brothers (table $1, \mathrm{~A}=1$, $\mathrm{X}=0, \mathrm{G}=2$ ), $1.3 \%$. If prenatal information is available the risk of the appropriate chromosome can be doubled.

Linkage programs currently do not routinely allow for germline mosaicism. This effect may be reduced by allowing for a rather high mutation rate. ${ }^{6}$ Grimm et al ${ }^{9}$ described an approach to estimating parameters at the level of the population. This results in much easier algebra and could therefore be incorporated into existing computer programs like LINKAGE. ${ }^{11}$ Jeanpierre $^{12}$ devised a computer program to calculate the probability of a possible carrier, in order to settle the origin of a mutation of a given family.

This work is supported by the Netherlands Organisation for Scientific Research.

Appendix

In the derivation of the formula presented in this paper, the same assumptions are made as $\mathrm{Hartl}^{2}$ did for the synchronous, symmetric, dichotomous model. Therefore, in the Bayesian formula all terms are the same as derived by Hartl, ${ }^{2}$ except the term $\mathrm{P}(\mathrm{F} \mid \mathrm{M}=\mathrm{i})$. This term has to be extended with the third informative class: the typed unaffected sibs with the at risk chromosome (class $\mathrm{C}$, with c sibs).

$$
P(F \mid M=i)=K \cdot \frac{1}{2}^{(i+1) \cdot a} \cdot\left(1-\frac{1}{2}^{(i+1)}\right)^{b} \cdot\left(\frac{1}{2}^{-\frac{1}{2}}{ }^{(i+1)}\right)^{c} .
$$

As in the formula of $\mathrm{Hartl}^{2}$ the birth order $\mathrm{K}$ of the sibs in the sibship is irrelevant, because this term appears in the Bayes formula in both the numerator and denominator and therefore cancels out. Note that the number of unaffected sibs (b) in this extended formula are the untyped unaffected sibs only. The number of affected sibs (a) stays the same as in the original formula. Substituting the term above and the terms derived by $\mathrm{Hartl}^{2}$ into the Bayes formula leads to the recurrence risk formula $P(A \mid F)$ as presented in this paper.

1 Murphy EA, Cramer DW, Kryscio RJ, Brown CC, Pierce ER. Gonadal mosaicism and genetic counseling for $\mathrm{X}$ linked recessive lethals. Am f Hum Genet 1974;26:207-22. Genet 1971;23:124-34.

Genet 1971;23:124-34.
3 Wijsman EM. Recurrence risk of a new dominant mutation Wijsman EM. Recurrence risk of a new dominant mutation
in children of unaffected parents. Am $\mathcal{f}$ Hum Genet 1991; 48:654-61.

4 Edwards JH. Familiarity, recessivity and germline mosaicism. Ann Hum Genet 1989;53:33-47.

5 Bakker E, Veenema H, Den Dunnen JT, et al. Germinal mosaicism increases the recurrence risk for 'new' Duchenne muscular dystrophy mutations. $₹$ Med Genet 1989 ; 26:553-9.

6 Ott J. Analysis of human genetic linkage. Revised ed. Baltimore: The Johns Hopkins University Press, 1991

7 Van Essen J, Abbs S, Baiget M, et al. Parental origin and germline mosaicism of deletions and duplications of the dystrophin

8 Bakker E, Van Broeckhoven C, Bonten EJ, et al. Germline mosaicism and Duchenne muscular dystrophy mutations. Nature 1987;329:554-6.

9 Grimm T, Muller B, Muller CR, Janka M. Theoretical considerations on germline mosaicism in Duchenne muscular dystrophy. F Med Genet 1990;27:683-7.

10 Passos-Bueno MR, Bakker E, Kneppers ALJ, et al. Different mosaicism frequencies for proximal and distal Duchenne muscular dystrophy (DMD) mutations indicate difference in etiology and recurrence risk. Am $\mathcal{F}$ Hum Genet 1992; 51:1150-5.

11 Lathrop GM, Lalouel JM. Easy calculation of lod scores and genetic risks on small computers. Am $\mathcal{f}$ Hum Genet 1984;36:460-5.

12 Jeanpierre $M$. Germinal mosaicism and risk calculation in $\mathrm{X}$ linked diseases. Am $\mathcal{f}$ Hum Genet 1992;50:960-7. 